\title{
Human Identification from Walking Signal based on Measurement of Current Generated by Electrostatic Induction
}

\author{
Koichi KURITA \\ Faculty of Engineering, Kinki University, 1 Takaya-Umenobe, Higashi-hiroshima-shi, Hiroshima 739-2116, Japan
}

\begin{abstract}
We have presented a novel method for human gait recognition, which is based on detecting the electrostatic induction current generated by the walking motion under non-contact conditions. The method involves the measurement of this electrostatic induction current, which flows through a measurement electrode. A model for the electrostatic induction current generated because of a change in the electric potential of the human body has been proposed. This model effectively explains the behavior of the waveform of the electrostatic induction current flowing through the electrode. Walking waveforms of 29 healthy individuals aged between 12 and 53 years were obtained. All the subjects wore rubber-soled shoes during the experiment. After Fourier analysis of the obtained waveform, the differentiated waveform of the gait spectrum was obtained in order to derive the subtle characteristics from the gait spectrum. The Pearson correlation coefficients with each other were obtained by data processing using methods such as differentiation and normalization. Results show that there is poor correlation between the walking waveforms. This suggests that the proposed technique based on the detection of subtle differences in the walking signal can be successfully applied for human identification.

Keywords: Walking Motion, Non-contact Measurement, Human Identification, Pearson correlation coefficients
\end{abstract}

\section{INTRODUCTION}

Biometric characteristics can be broadly classified into two main categories-physiological and behavioral. Physiological characteristics are associated with the shape of the body in a broad sense. Examples of physiological biometrics include fingerprint [1], palmar print [2], hand geometry [3], vein authentication [4], ear recognition [5], face recognition [6], iris recognition [7], retina recognition [8], odor recognition [9], and DNA as the shape of the information about the human genome. On the other hand, behavioral characteristics are related to the behavior of a person. Examples of behavioral biometrics include typing rhythm, gait, and voice.

Fingerprint, iris, and vein authentications have been successfully employed in automatic human identification systems. However, these systems require the subject's cooperation such as the action of placing the subject's hand on the sensor because the resolutions of raw pictures usually influence the recognition rate. Therefore, these systems require the subject to be close to the sensor head for accurate identification.

On the other hand, researchers have made numerous attempts to develop methods for behavioral biometrics, because behavioral biometrics has unique advantages over conventional physiological biometrics. In particular, gait recognition may have great potential for not only human identification but also human emotion discrimination.
Since the human walking motion results in a self-sustaining and dynamic rhythm owing to the integrated signals generated from the spinal cord and sensory feedback, it contains unique characteristics of each individual.

In the last decade, gait analysis for human recognition has attracted much attention from computer vision researchers motivated by its wide applications [10-20]. Normally, in vision-based gait recognition, motion features are extracted from image sequences. Subsequently, identification process by comparing between feature amounts is executed. However, in conventional gait recognition methods, the experimental conditions such as the distance between the subject and the camera, the angle of the camera, and the pixel density of the camera significantly influence the recognition rate of the system. Moreover, the main drawback of these methods is that they cannot detect anything in the dead zone of the camera. Furthermore, many image-processing systems require a complex logic to ignore the disturbances caused by the motion of objects other than humans.

Alternatively, body-mounted accelerometers [21, 22] are extensively used for monitoring human motion in gait analysis because these systems are inexpensive as compared with an optoelectronic motion capture system. Another advantage of body-mounted accelerometers is that the system is available in indoor-outdoor space. As an alternative, in-shoe pressure measurement systems are useful for gait analysis to obtain the plantar pressure and force data during dynamic walking motion [23]. The 
advantage of this system is that the subject can move freely if he/she is fitted with a data logger. Additionally, several methods have been presented to measure gait parameters by using several techniques, e.g., an ultrasonic motion analysis system for measurement of temporal and spatial gait parameters [24], an electromagnetic 3D orientation estimation system that uses the earth's magnetic field [25], and a wearable ultrasonic motion analysis system. However, these measurement systems are necessary to contact the some kind of sensor or maker to subject. Therefore, these methods as referred to above have not been applied to the non-contact detection for gait analysis. Naturally, these methods can not apply to the gait analysis for human recognition. If an effective non-contact, non-attached human motion measurement system for nearly unlimited use anywhere is developed, this system is expected to have a wide field of application, e.g., not only in human identification but also in ambulatory measurement during daily activities, clinical gait analysis, and rehabilitation aid system in clinical practice, etc.

In this paper, we have presented a new direction for gait recognition without the use of a camera or video images. We have developed an effective non-contact technique for the detection of human walking motion using human-generated body charge. This technique involves the detection of an electrostatic induction current of the order of approximately sub-picoamperes flowing through an electrode that is placed at a distance of $3 \mathrm{~m}$ from a subject. The absolute value of the electrostatic induction current depends on the type of footwear and floor material. However, we confirmed that this technique detects the electrostatic induction current generated by the walking motion in daily life, because the proposed technique allows ultrasensitive detection of the electrostatic induction current. This technique effectively explains the behavior of the waveform of the electrostatic induction current flowing through a given measurement electrode through a capacitance model for the human body. Gait waveforms of 29 healthy individuals aged between 12 and 53 years were obtained by the proposed technique. As a pretreatment step, Fourier analysis was performed to obtain the gait spectrum. Subsequently, the differentiated waveform of the gait spectrum was obtained in order to derive the subtle characteristics from the gait spectrum. The Pearson correlation coefficients with each other of the differentiated waveform of the gait spectrum were obtained. Results show that there is poor correlation between each waveform. This suggests that human identification based on the subtle differences in the walking signal can be realized using the proposed technique.

\section{PRINCIPLE}

The human body is electrically charged during walking [26-30]. In the case of a subject standing or walking, we assume that there are two highly resistive layers between the feet of the subject and the floor, as shown in Figure 1. One layer is the sole of the subject's footwear. The other is the surface of the floor. Capacitance $C_{s f}$ of the feet relative to the ground may be calculated as the sum of capacitance $C_{s}$ of the sole and capacitance $C_{f}$ of the floor surface. In addition, $C_{0}$ is the capacitance of the rest of the subject's body relative to nearby objects on the floor. Therefore, potential $U_{B}$ of the human body when it is in walking motion can be expressed as follows [28].

$$
U_{B}=Q_{B} \frac{\varepsilon_{a} S+x C_{B}}{C_{B} \varepsilon_{a} S},
$$

where

$$
C_{B}=C_{0}+C_{s f}=C_{0}+\frac{C_{s} C_{f}}{C_{s}+C_{f}} .
$$

$Q_{B}$ is the instantaneous charge of the human body during walking motion, $\varepsilon_{a}$ is the permittivity of the air gap between the sole and the floor, and $S$ is the effective sole area at height $x$ above the floor. The induced charge $Q$ of the measurement electrode placed at a certain distance from the subject can be expressed as follows:

$$
Q=C\left(U_{B}-V\right),
$$

where $C$ is the capacitance between the human body and the measurement electrode, and $V$ is the potential of the measurement electrode. From the above two equations, the induced current $I$ flowing through the measurement electrode can be expressed as follows:

$$
I=\frac{d Q}{d t}=C \frac{d U_{B}}{d t}=C Q_{B}\left(-\frac{x}{\varepsilon_{a} S^{2}} \frac{d S}{d t}+\frac{1}{\varepsilon_{a} S} \frac{d x}{d t}\right) .
$$

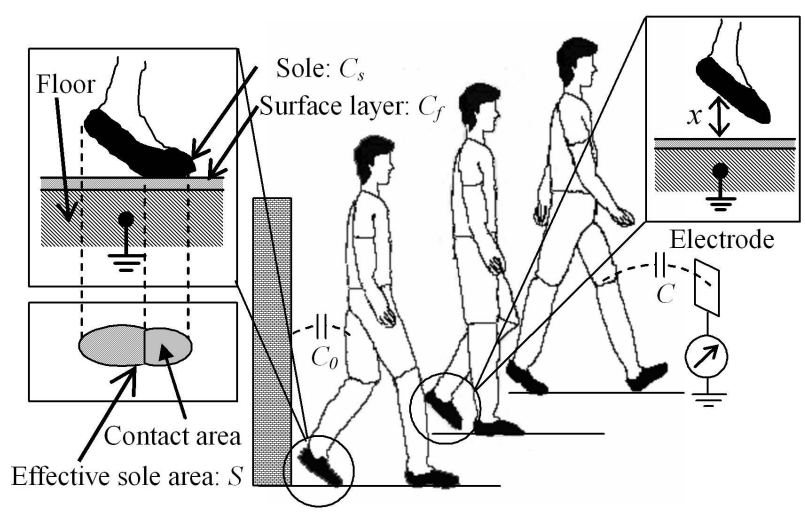

Figure 1: Schematic of interaction between human body and floor surface. 
We assume that the human body is a good conductor. The first term in Eq. (4) represents the current induced by the motion of the foot before it is lifted off the floor. The second term represents the current induced by the motion of the foot and leg after the foot is lifted off the floor. The second term is approximately proportional to the velocity of the foot. Therefore, in the case of walking motion near the measurement electrode, it is possible to measure the current generated under perfect non-contact conditions.

\section{EXPERIMENTAL METHOD}

\subsection{Measurement of gait spectrum}

The schematic of the measurement system for detecting the very weak electric current generated by the stepping motion is shown in Figure 2. The electrostatic induction current flowing through the electrode placed at a distance of $3 \mathrm{~m}$ from the subject was converted into voltage using an $I-V$ converter comprising an operational amplifier. The conversion ratio of the $I-V$ converter was $3 \mathrm{~V} / \mathrm{pA}$. As shown in Fig. 3, the $I$ - $V$ converter consists of two low-input-current op-amps. The selected low-noise op-amp has an input offset voltage of $40 \mu \mathrm{V}$ and input offset current of $1 \mathrm{pA}$. The feedback resistor connected to the op-amp is a hermetically sealed high register that can prevent stray current due to humidity. For such measurements with high input resis-

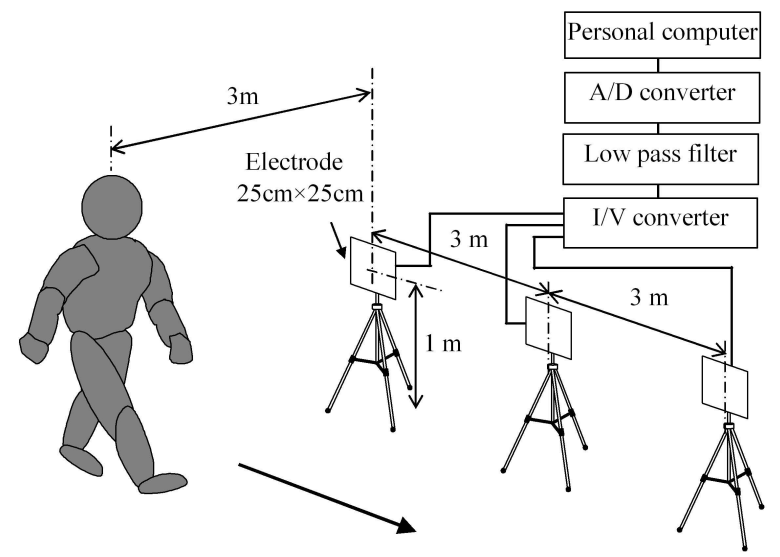

Figure 2: Schematic of system for measuring electrostatic induction current generated by human walking motion.

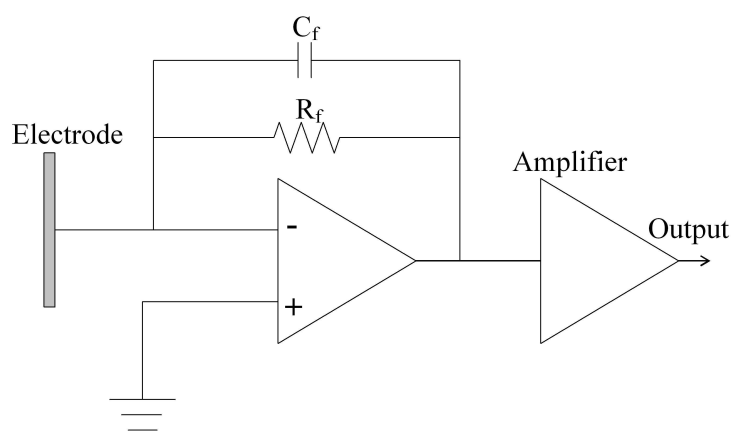

Figure 3: Schematic circuit diagram of the $I-V$ converter. tance, a conventional guarding method is absolutely imperative for shielding used with op-amps; this prevents stray currents from entering sensitive nodes. Sensitive nodes are completely surrounded by a guard conductor that is kept at the same electric potential as the sensitive node. A very weak electric current of the order of sub-picoamperes was measured. The induction current generated by the commercial power source used in the experiment appears as noise in the measured electrostatic induction current. To remove this, we use a filtering system with a cutoff frequency of $20 \mathrm{~Hz}$. Therefore, the measurement system is unaffected by the noise from other electronic devices such as mobile phones or microwave ovens. The static electric field generated by the presence of charged material around the measurement electrode affects the absolute value of capacitance $C$, which is formed between the subject's body and a given measurement electrode. However, the static electric field has little effect on the measurement, because the instantaneous electrostatic induction current arises from the change in capacitance $C$ according to the subject's walking motion. In fact, the transient current generated can be detected only when the subject's body is in motion.

The analog signals were subsequently converted into digital signals by using an A/D converter, and the obtained data were stored in a personal computer at a sampling frequency of $250 \mathrm{~Hz}$. A detection electrode with an area of $625 \mathrm{~cm}^{2}$ was placed $1 \mathrm{~m}$ above the floor and $3 \mathrm{~m}$ away from the center of the subject.

Gait waveforms of 29 healthy individuals aged between 12 and 53 years were obtained using the proposed technique. All the subjects wore rubber-soled shoes during the experiment. The floor was made of PVC (polyvinyl chloride). We performed the measurements at a temperature of $23^{\circ} \mathrm{C}$ and a relative humidity of $84 \%$. In general, the strength of the electrostatic induction current generated due to walking motion was not affected by the experimental factor such as room temperature, a subject's clothes, and socks. However, the strength of the electrostatic induction current generated differ according to the surface roughness of the floor, humidity, and the type of shoes. The waveform of the electrostatic induction current is dependent on the type of shoes used by the subject because the effective sole area $S$ is different for different shoes. Therefore, to ensure that the obtained waveforms of the electrostatic induction current are reproducible, the same type of shoes should be used during the experiments. While we use the same type of shoes, obtained waveforms of the electrostatic induction current are used as data of the interpersonal comparison and of the temporal change. The subjects were asked to walk naturally at the site of the experiment. The subjects were asked to walk naturally along the 7-meter path at the site of the experiment. Each individual provides 10 walking wave- 
forms. The intensity of the electrostatic induction current was independent of the angle between the electrode and the subjects facing the electrode; it was inversely proportional to the distance between the subject and the electrode. The proposed technique showed good reproducibility.

\subsection{Data processing}

The obtained waveform of the electrostatic induction current contains a component of the gait cycle. In the normal walking motion of an adult male, it is said that one complete gait cycle is approximately $0.55 \mathrm{~s}$. Therefore, the obtained waveforms are strongly periodically modulated by these gait cycles, which resemble each other of subject. Therefore, as the first step, the spectrum of the waveform of the electrostatic induction current was obtained by Fourier analysis. A peak generated by one complete gait cycle is observed around $1.8 \mathrm{~Hz}$ in each spectrum. The frequency of this peak that occurs because of one complete gait cycle in each spectrum was normalized at $1.8 \mathrm{~Hz}$ by interpolation of the spectrum in order to remove the difference of one complete gait cycle for each individual.

Furthermore, the waveform of the gait spectrum differentiated with respect to frequency is obtained in order to emphasize the subtle individual characteristics from the entire gait spectrum. Thus, we can estimate the subtle differences in the walking style can be detected using the differential waveform with respect to frequency. The differentiated waveform from $0 \mathrm{~Hz}$ to $20 \mathrm{~Hz}$ was treated as the feature amount for each individual. Finally, in order to investigate the possibility of human identification, the feature amounts of each individual were compared. The Pearson correlation coefficients of the same person was calculated using 2 walking waveforms drawn at random from 10 walking waveforms. Between each person, the Pearson correlation coefficients with each other of the future amount were obtained. Figure 4 shows the overview of the proposed data processing method.

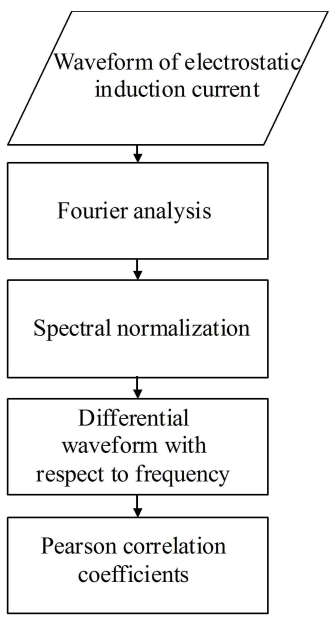

Figure 4: Overview of the proposed data processing method.

\section{RESULTS AND DISCUSSIONS}

Figure 5 shows the typical waveform of the current generated by the human motion of walking. Cadence components are observed in the resulting waveform for each case. These components indicate the presence of a gait cycle in the walking motion. The gait cycle consists of a combination of alternating swing and stance phases of the left and right foot.

The waveform contains cadence components of both the feet during bipedal walking; this reveals that the toe of the left foot is lifted off the floor and simultaneously the heel of the right foot comes into contact with the floor. When the toe of the right foot is lifted off the floor, the effective sole area $S$ decreases and distance $x$ between the right foot and the floor increases continuously. As a result of the walking motion, current $I$ flowing through the measurement electrode increases, as predicted by the first term on the right-hand side of Eq. (4). In rapid succession, $I$ decreases, as predicted by the second term on the righthand side of Eq. (4). Furthermore, in the second half of the swing phase, a rapid decrease in $x$ induces a decrease in $I$, as predicted by the second term on the right-hand side of Eq. (4). In rapid succession, I decreases with an increase in the effective sole area $S$ resulting from the heel contact, as predicted by the first term on the right-hand side of Eq. (4). Therefore, Eq. (4) effectively explains the behavior of the waveform of the electrostatic induction current $I$ flowing through the measurement electrode.

Figure 6 shows the typical FFT-analyzed spectrum data of the electrostatic induction current waveform generated by the walking motion. The FFT spectrum shows a peak at approximately $1.8 \mathrm{~Hz}$. It is obvious that this peak is attributed to the presence of the gait cycle. In this figure, it can be clearly observed that one primary and few harmonic peaks are induced by the walking cycle.

Figure 7 shows the histogram of the Pearson correlation coefficients of 841 with each other of the future amount

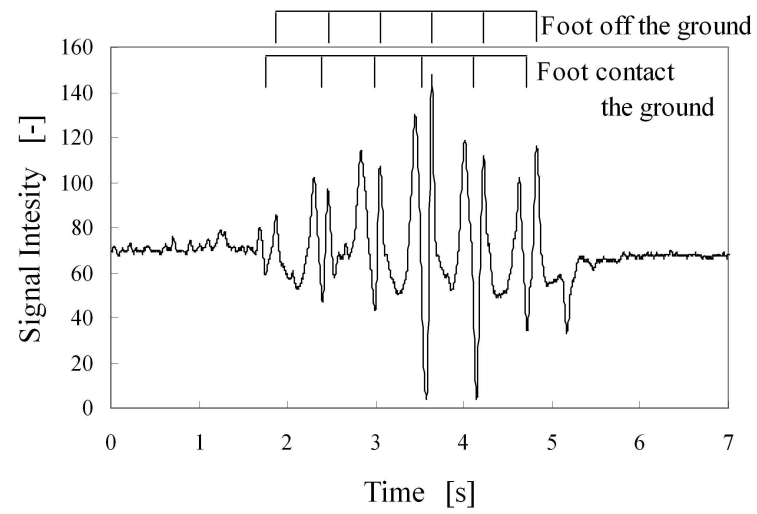

Figure 5: Typical waveform of current generated by human walking motion. 


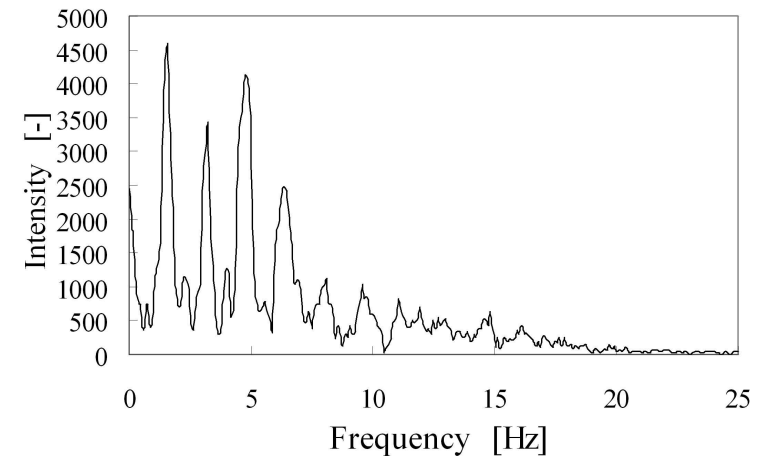

Figure 6: Typical FFT-analyzed spectrum data of electrostatic induction current generated during human walking motion.

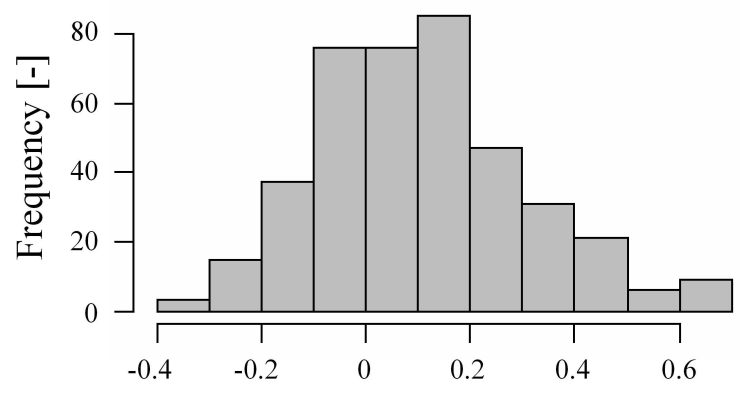

Pearson correlation coefficients [-]

Figure 7: Histogram of the Pearson correlation coefficients with each other of the future amount.

of 29 subjects. The correlation coefficient ranges from -1 to 1 . Values 1 and -1 imply a positive and negative correlation between the variables, respectively. Value 0 implies that there is no correlation between the variables. Several authors have proposed guidelines for the interpretation of a correlation coefficient. According to these guidelines, a correlation coefficient between -0.09 and 0.09 implies no correlation. A correlation coefficient between -0.1 and -0.3 and between 0.1 and 0.3 implies small correlation. The obtained histogram shows a peak at a correlation coefficient of approximately 0.1 . The standard deviation of the correlation coefficient is 0.3 . Results show that there is poor correlation between each future amount. Figure 8 shows the absolute value of the correlation coefficient as a graduation image for the 29 subjects. This figure shows the discrimination capability of the proposed technique.

Moreover, we constructed a walking signal database which contains 290 walking waveforms, for the purpose of testing the performance of the proposed technique. These waveforms are from 29 different persons. Each individual provides 10 walking waveforms.

In general, false acceptance rate (FAR) means the probability that the system incorrectly matches the input data to a non-matching template in the database. On the other

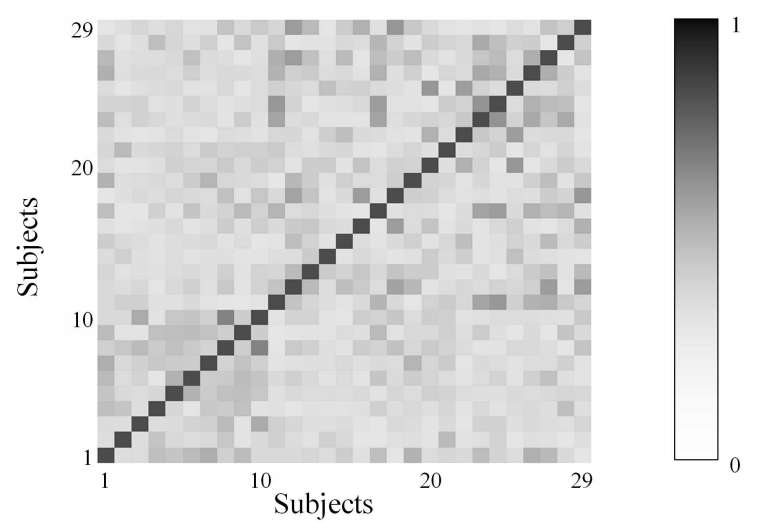

Figure 8: Cluster separation of various feature amounts.

hand, false rejection rate (FRR) means the probability that the system fails to detect a match between the input data and a matching template in the database. Here, FAR and FRR mean the error of the first kind (type I error) and the error of the second kind (type II error), respectively. When the threshold value of Pearson correlation coefficients is 0.7 , we obtain the identification result as shown in Table 1. An identification performance of about $0.61 \%$ FAR and about $2.31 \%$ FRR have been obtained.

We can almost certainly say that human identification based on subtle differences in the walking signal can be realized using the proposed technique. It is generally known that the human motions of walking result in a selfsustaining and dynamic rhythm owing to the integrated signals generated from the spinal cord and the sensory feedback. The obtained results are in agreement with this knowledge.

\section{CONCLUSIONS}

In this paper, we have presented a new direction for gait recognition without the use of a camera or video images. The change in the electric potential of the human body that is caused by the walking motion induces an electrostatic induction current in the electrode placed at a distance of a few meters from the human body. Using this technology, we have developed an effective non-contact technique for the detection of human walking motion by detecting the change in this human-generated body charge. This

Table 1: Result of the identification.

\begin{tabular}{|c|c|c|}
\hline $\begin{array}{c}\text { Threshold value of Pearson } \\
\text { correlation coefficients }\end{array}$ & FAR[\%] & FRR[\%] \\
\hline 0.60 & 5.76 & 0.11 \\
\hline 0.65 & 2.26 & 0.37 \\
\hline 0.70 & 0.61 & 2.31 \\
\hline 0.75 & 0.35 & 6.42 \\
\hline 0.80 & 0.21 & 12.3 \\
\hline
\end{tabular}


technique involves the detection of an electrostatic induction current of the order of approximately sub-picoamperes flowing through an electrode that is placed at a distance of $3 \mathrm{~m}$ from the subject. This technique effectively explains the behavior of the waveform of the electrostatic induction current flowing through a given measurement electrode through a capacitance model of the human body. Gait waveforms of 29 healthy individuals aged between 12 and 53 years were obtained by the proposed technique. By data processing techniques such as differentiation and normalization after Fourier analysis of the obtained waveform, we obtained the Pearson correlation coefficients with each other. Results show that there is poor correlation between each waveform. This suggests that human identification based on subtle differences in the walking signal can be achieved using the proposed technique. In our future work, we intend to improve the data processing method to realize real-time recognition. Furthermore, more walking waveforms must be collected and over a longer period of time.

\section{ACKNOWLEDGEMENT}

This research was partially supported by the Grant-inAid for Scientific Research (B), 23310117, 2012.

\section{REFERENCES}

1. K. Karu and A. K. Jain, "Fingerprint classification", Pattern Recognition, 29, (3), pp.389-404 (1996).

2. A. Kong, D. Zhang, and M. Kamel, "A survey of palmprint recognition”, Pattern Recognition, 42, (7), pp.1408-1418 (2009).

3. N. Duta, "A survey of biometric technology based on hand shape", Pattern Recognition, 42, (11), pp.27972806 (2009).

4. D. Mulyono, and H. S. Jinn, "Astudy of finger vein biometric for personal identification", International Symposium on Biometrics and Security Technologies 2008, pp.1-8 (2008).

5. L. Nanni, and A. Lumini, "Fusion of color spaces for ear authentication", Pattern Recognition, 42, (9), pp.1906-1913 (2009).

6. R. Chellappa, C. Wilson, and S. Sirohev, "Human and machine recognition of faces: a survey", Proceedings of IEEE. 83, (5), pp.705-740 (1995).

7. H. A. Park, and K. R. Park, "Iris recognition based on score level fusion by using SVM", Pattern Recognition Letters, 28, (15), pp.2019-2028 (2007).

8. S. Sanderson, and J. Erbetta, "Authentication for secure environments based on iris scanning technology", IEE Colloquium on Visual Biometrics, pp.8/1-8/7 (2000).
9. D. Malaspina, E. Coleman, R. R. Goetz, J, HarkavyFriedman, C. Corcoran, X. Amador, S. Yale, and J. M. Gorman, "Odor identification, eye tracking and deficit syndrome schizophrenia”, Biological Psychiatry, 51, (10), pp.809-815 (2002).

10. J. B. Hayfron-Acquah, M. S. Nixon, and J. N. Carter, "Automatic gait recognition by symmetry analysis", Pattern Recognition Letters, 24, (13), pp.2175-2183 (2003).

11. P. S. Huang, C. J. Harris, and M. S. Nixon, "Human gait recognition in canonical space using temporal templates", IEE Proceedings_-Vision, Image and Signal Processing, 2, (146), pp.93-100 (1999).

12. S. V. Stevenage, M. S. Nixon, and K. Vince, "Visual analysis of gait as a cue to identity", Applied Cognitive Psychology, 13, pp.513-526 (1999).

13. L. Lee, and W. E. L. Grimson, "Gait analysis for recognition and classification", Proceedings of the IEEE Conference on Face and Gesture Recognition, pp.155-161 (2002).

14. C. Y. Yam, M. S. Nixon, and J. N. Carter, "Automated person recognition by walking and running via modelbased approaches", Pattern Recognition, 37, (5), pp.1057-1072 (2004).

15. A. Kale, et al., "Identification of humans using gait", IEEE Transactions on Image Processing, 13, (9), pp.1163-1173 (2004).

16. J. Han, and B. Bhanu, "Individual recognition using gait energy image", IEEE Transactions on Pattern Analysis and Machine Intelligence, 28, (2), pp.316322 (2006).

17. L. Wang, T. Tan, W. Hu, and H. Ning, "Automatic gait recognition based on statistical shape analysis", IEEE Trans. Image Process, 12, pp.1120-1131 (2003).

18. T. Lam, R. Lee, and D. Zhang, "Human gait recognition by the fusion of motion and static spatio-temporal templates", Pattern Recognition, 40, (9), pp.25632573 (2007).

19. P. S. Huang, C. J. Harris, and M. S. Nixon, "Human gait recognition in canonical space using temporal templates", IEE Proceedings-Vision, Image, and Signal Processing, 146, (2), pp.93-100 (1999).

20. J. P. Foster, M. S. Nixon, and A. Prügel-Bennett, "Automatic gait recognition using area-based metrics", Pattern Recognition Letters, 24, (14), pp.2489-2497 (2003).

21. J. R. W. Morris, "Accelerometry a technique for the measurement of human body movements", Journal of Biomechanics, 6, pp.729-739 (1973).

22. R. Williamson, and B. J. Andrews, "Detecting absolute human knee angle and angular velocity using accelerometers and rate gyroscopes", Med. Biol. Eng. 
Comput., 39, pp.1-9 (2001).

23. T. C. Pataky, P. Caravaggi, R. Savage, and R. H. Crompton, "Regional peak plantar pressures are highly sensitive to region boundary definitions", Journal of Biomechanics, 41, pp.2772-2775 (2008).

24. R. B. Huitema, A. L. Hof, and K. Postema, "Ultrasonic motion analysis system-measurement of temporal and spatial gait parameters", Journal of Biomechanics, 35, pp.837-842 (2002).

25. K. Kobayashi, L. Gransberg, E. Knutsson, and P. Nolen, "A new system for three-dimensional gait recording using electromagnetic tracking", Gait\& Posture, 6, pp.63-75 (1997).

26. K. Takiguchi, T. Wada, and S. Toyama, "Human Body Detection that Uses Electric Field by Walking", Journal of Advanced Mechanical Design, Systems, and Manufacturing, 1, (3), pp.294-305 (2007).

27. V.Amoruso, M. Helali, and F. Lattarulo, "An Improved Model of Man for ESD Application", Journal of Electrostatics, 49, pp.225-244 (2000).

28. T. Ficker, "Electrification of Human Body by Walking", Journal of Electrostatics, 64, pp.10-16 (2006).
29. Koichi Kurita, "New Estimation Method for the Electric Potential of the Human Body under Perfect Noncontact Conditions", IEEJ Trans. on Elect. and Electronic Engi., 4, pp.309-311 (2009).

30. Koichi Kurita, and Shinya Ueta, "New Motion Control Method for Bipedal Robot Based on Noncontact and Nonattached Human Motion Sensing Technique", IEEE Transactions on Industry Applications, 47, (2), pp.1022-1027 (2011).

\section{Koichi KURITA}

Koichi Kurita was born in Hokkaido Japan, on December 11, 1958. He received the B.E. degree in applied physics from Tokyo Science University, Tokyo, Japan, in 1981, and M.E. and D.E. degrees in material engineering from Hiroshima University, Hiroshima, Japan, in 1983 and in 1987, respectively. From 1988 to 2007 , he worked in the R\&D Center, Mitsubishi Heavy Industry Ltd., Hiroshima, Japan. Now, he is a Professor of Department of Electronic Engineering and Computer Science at Faculty of Engineering, Kinki University since 2012, Hiroshima Japan, after having contributed for 5 years as a Professor of Electrical Engineering and Information Science at Kochi National College of Technology since 2007, Kochi Japan. He has been working on development of noncontact sensing systems. 